\title{
Tunneled island flaps in facial defects reconstruction*
}

\author{
Miguel Pinto de Gouveia ${ }^{1}$ \\ Ana Brinca ${ }^{3}$
}

\author{
Ana Isabel Gouveia ${ }^{2}$ \\ Ricardo Vieira ${ }^{3}$
}

DOI: http:/ /dx.doi.org/10.1590/abd1806-4841.20175565

\begin{abstract}
The reconstruction of facial surgical defects is usually challenging for the dermatologic surgeon. Three different cases of facial defects in which tunneled island flaps were used are reported. In 2 cases, wide defects involving the nasal dorsum and ala were repaired using a nasolabial island flap tunneled through the lateral side of the nose. A tunneled island glabellar flap was used for medial canthus reconstruction in the third case. Despite complex pedicle dissection and frequent trapdoor deformation, tunneled island flaps allow reconstruction of wide defects in a single-staged procedure, camouflaging the scar of the donor area in boundaries of cosmetic units and preserving the facial central symmetry.
\end{abstract}

Keywords: Face; Surgical flaps; Reconstruction

\section{INTRODUCTION}

Facial surgical defects are challenging for the dermatologic surgeon because of the aesthetic importance and low reserves of adjacent skin in some cosmetic units.

In order to face these difficulties, tunneled island flaps, a variant of classic island flaps, have been used in facial reconstructive surgery, particularly in deep defects of the eye's inner canthus, and the dorsum of the nose and alae.

While performing a tunneled flap, a cutaneous island with dimensions similar to the defect is created, near but not adjacent to it. The incision encompasses the whole circumference of the flap on the surface, but preserves a subcutaneous pedicle, with enough length to allow mobilizing the cutaneous island towards the defect, through a subcutaneous tunnel created between the donor and receptor areas. this flap.

\section{CASE REPORT}

\section{Case 1}

The radical excision of a squamous cell carcinoma (SCC) from the dorsum of the nose resulted in a wide defect involving the lateral wall of the nose and part of the ala nasi. The reconstruction was performed using a nasolabial island flap with the dissection of a subcutaneous pedicle up to the medial canthus of the eye and then tunneled along the lateral wall of the nose. Fifteen months after the procedure there were no signs of recurrence of the SCC and the final result was considered satisfactory by the patient and by the surgical team (Figure 1).

\section{Case 2}

The radical excision of a basal cell carcinoma (BCC) resulted in a wide defect involving the tip, dorsum, and left ala of the nose. The reconstruction was performed using an island flap designed on the nasolabial fold, with a tunneled subcutaneous pedicle through

Work Submitted on 10.01.2016

Approved by the Advisory Board and accepted for publication on 27.04.2016

* Study conducted at the Dermatology Department of the Centro Hospitalar e Universitário de Coimbra (CHUC) - Coimbra, Portugal.

Financial support: None

Conflict of interest: None

Department of Dermatology at Centro Hospitalar e Universitário de Coimbra (CHUC) - Coimbra, Portugal.

Department of Dermatology at Centro Hospitalar Lisboa Norte, EPE (CHLN) - Lisboa, Portugal.

Dermatologic Surgery Unit at Centro Hospitalar Universitário de Coimbra (CHUC) - Coimbra, Portugal.

(C)2017 by Anais Brasileiros de Dermatologia 
the right ala nasi. After nine months of follow-up, there were no signs of recurrence of the BCC. A significant trapdoor effect could be seen, despite the frequent massaging of the flap, that improved after intralesional corticosteroid (Figure 2).

\section{Case 3}

The radical excision of a BCC from the left medial canthus of the eye resulted in a deep defect in that area. To cover it, we resorted to a glabellar island flap with a subcutaneous pedicle dissected inferiorly, that was then mobilized through a subcutaneous tunnel created in the glabella. After 14 months of follow-up there were no signs are recurrence of the BCC. There was a mild trapdoor effect that, however, did not worry the patient (Figure 3).

\section{DISCUSSION}

Tunneled island flaps have been described for the reconstruction of the medial canthus of the eye, dorsum and ala of the nose, and when adequately performed, represent an excellent alternative. ${ }^{1-5}$ The tunneling principle has been applied in the repair of defects of other areas, such as upper lip, nasal vestibule, and the ear, the concha in particular (also known as flip-flop flap), and earlobe. ${ }^{3,6-8}$
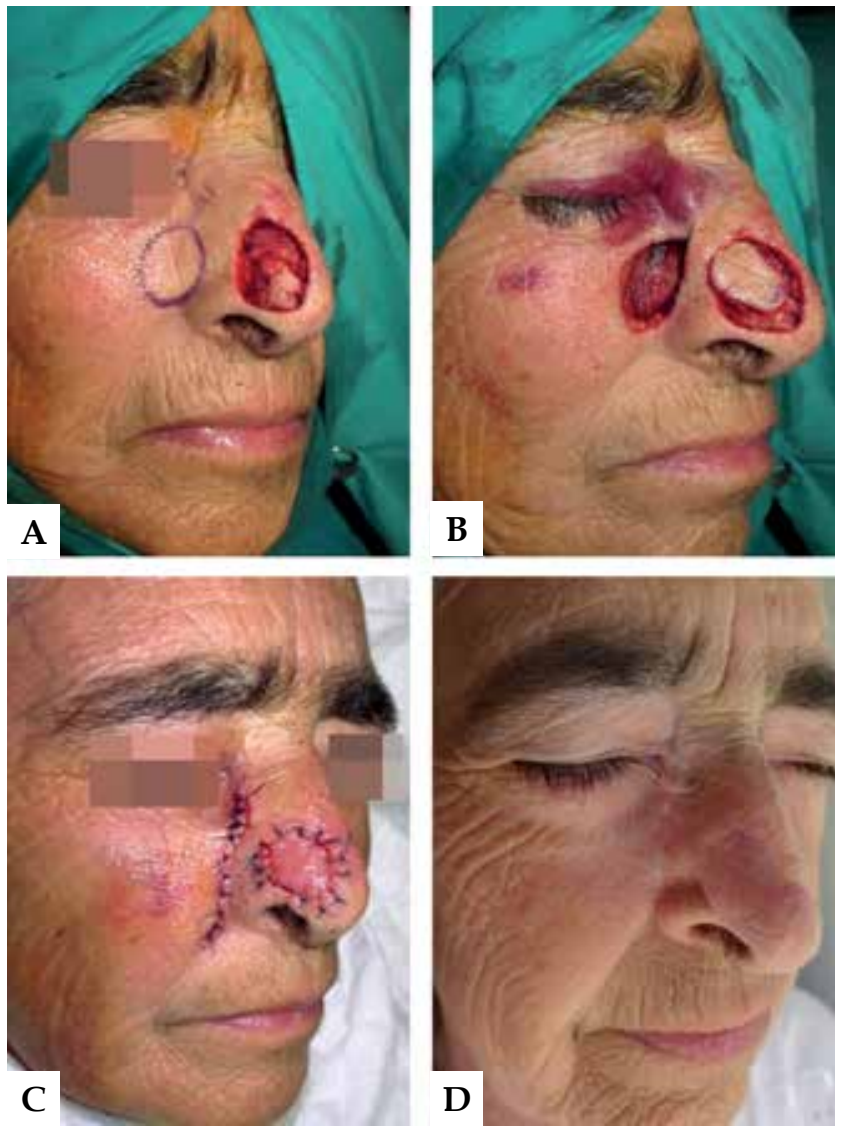

Figure 1: A: Defect involving the right side of the dorsum of the nose and part of the right nasal ala. B: Nasolabial island flap tunneled through the right side of the nose in order to reach the primary defect. C: Donor area scar in a transition area of cosmetic units. D: Fifteen months after surgery
Being a variant of island flaps, these flaps are intensely irrigated, extraordinarily mobile and the created tunnel allows a more direct course towards the primary defect..$^{3.9}$ Despite being technically demanding, they only require one stage, therefore eliminating the morbidity of further procedures.

These flaps must be considered for the defects of the dorsum and ala of the nose, particularly when the alar crease and the lateral nose are intact, in order to allow tunneling of the flap through these structures, such as in case 1 and 2. ${ }^{4}$ The design for this flap allows planning of the scar on the donor area to be placed in transition areas of cosmetic units, such as demonstrated in the cases 1 and 2 , where the scars were hidden in the nasolabial fold. $\frac{10}{}$ With this option, we were able to repair extensive defects of the nose, where tissue mobility is reduced, maintaining color and texture of the adjacent skin and preserving the alar crease.

Another advantage of these flaps is the ability to repair deep defects, namely the ones on the medial canthus of the eye, as demonstrated in case 3. Secondary intention healing is usually a good option in these cases, but for extensive and deep defects that need reconstruction, glabellar or frontal tunneled island flaps allow reconstruction of the concavity of the medial canthus. ${ }^{1}$
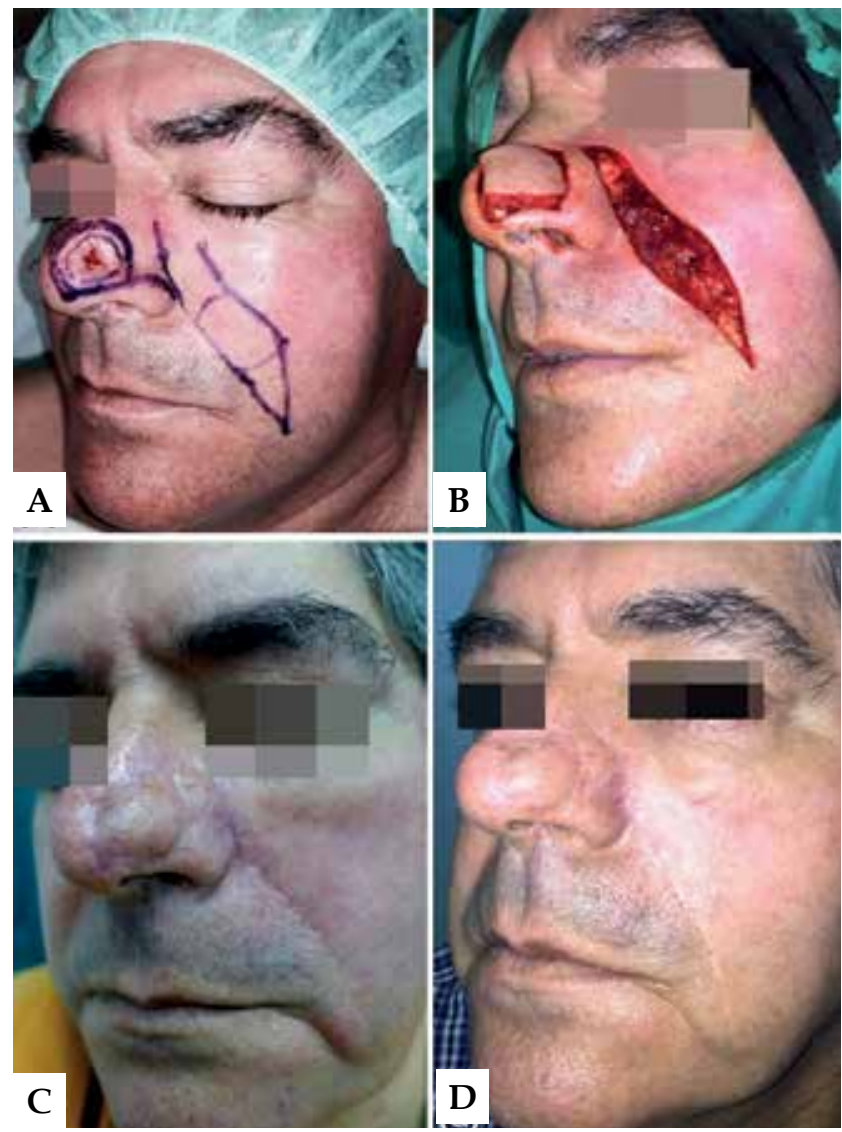

Figure 2: A: Island flap designed on the nasolabial fold. B: Subcutaneous flap dissected in the superior nasolabial area and then tunneled through the right nasal ala. C: Nine months after surgery, significant trapdoor effect. D: Improvement of the trapdoor effect three months after intralesional corticosteroid injection 

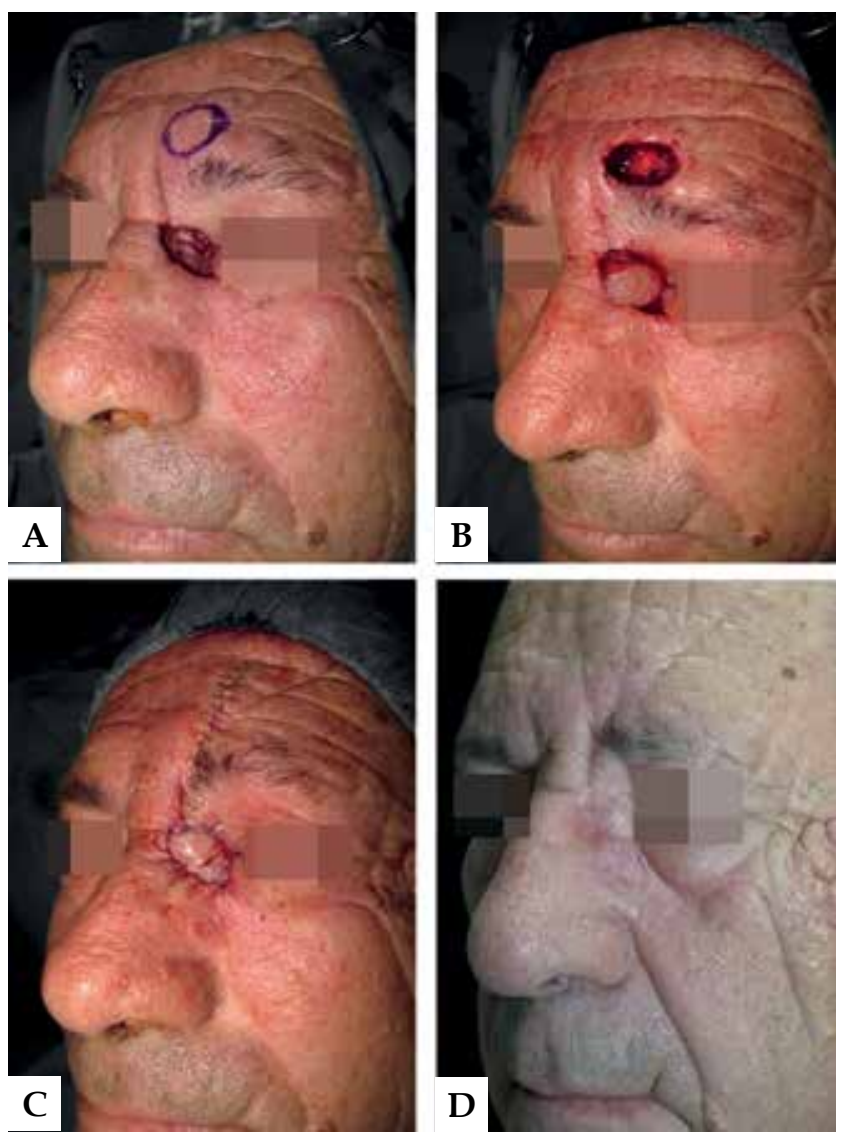

Figure 3: A: Deep defect on the medial canthus after radical excision of the tumor. B: Glabellar island flap mobilized through the subcutaneous tunnel created in the nasal bridge. C: Repair of the concavity of the medial canthus. D: Fourteen months after surgery

\section{REFERENCES}

1. Mombaerts I, Gillis A. The tunneled forehead flap in medial canthal and eyelid reconstruction. Dermatol Surg. 2010;36:1118-25

2. Karşdağ S, Sacak B, Bayraktaroglu S, Ozcan A, Ugurlu K, Bas L. A novel approach for the reconstruction of medial canthal and nasal dorsal defects: frontal hairline island flap. J Craniofac Surg. 2008;19:1653-7.

3. Cook JL. Tunneled and transposed island flaps in facial reconstructive surgery. Dermatol Surg. 2014;40:S16-29.

4. Kearney C, Sheridan A, Vinciullo C, Elliott T. A tunneled and turned-over nasolabial flap for reconstruction of full thickness nasal ala defects. Dermatol Surg. 2010;36:1319-24.

5. Krishnan RS, Clark DP, Donnelly HB. Tunneled transposition flap for reconstruction of defects of the nasal ala. Dermatol Surg. 2007;33:1496-501.

6. Kishi K, Imanishi N, Shimizu Y, Shimizu R, Okabe K, Nakajima H. Alternative 1-step nasal reconstruction technique. Arch Facial Plast Surg. 2012;14:116-21.

7. Fader DJ, Johnson TM. Ear reconstruction utilizing the subcutaneous island pedicle graft (flip-flop) flap. Dermatol Surg. 1999;25:94-6.

8. Wang SQ, Goldberg LH, Kimyai-Asadi A. Tunneled island pedicle flap for an earlobe defect. Dermatol Surg. 2007:33:835-7.

9. Georgiade NG, Mladick RA, Thorne FL. The nasolabial tunnel flap. Plast Reconstr Surg. 1969;43:463-6.

10. Mahlberg MJ. Tunneled melolabial pedicle flap for small but deep lateral alar rim ficial. Dermatol Surg. 2013;39:1527-9.
Tunneled island flaps are, however, technically difficult and the pedicle dissection is slow, thus, it has to be performed carefully.

It is necessary to measure correctly the pedicle to compensate the shortening caused while mobilizing and tunneling the flap, not damaging underlying structures in the process..$^{10}$ It is equally necessary to put the flap in position, in a way that blood flow is not compromised by tension or compression by the tunnel. Occasionally, there is a slight bulging in the tunneled pedicle, but this effect usually improves with time and rarely needs correction.

Besides the common risks to all surgical procedures, such as hemorrhage, infection and necrosis, these flaps are prone to the trapdoor effect, as seen in two of our patients (cases 2 and 3). The risk of this deformity can be minimized by deliberately reducing the flap area in 20 to $25 \% \underline{3}$ and undermining the borders of the receptor area. ${ }^{5}$

A subtle trapdoor effect does not require any action besides frequent massaging of the flap and it can actually be beneficial in some situations as, for example, when recreating the round shape of the ala nasi. 10

Other correction options include intralesional injection of corticosteroids, as we did for case 2 and, although more rarely needed, debridement of the flap, followed by Z plasty.

In sum, tunneled island flaps are a variant of traditional island flaps, that yield good results for the reconstruction of the medial canthus of the eye, dorsum and ala of the nose, despite the frequent trapdoor effect. They are an elegant way of repairing deep defects in complex anatomical locations, maintaining the central facial symmetry.

\author{
MAILING ADDRESS: \\ Miguel Pinto de Gouveia \\ Av. Bissaya Barreto \\ Praceta Prof. Mota Pinto \\ 3000-045 Coimbra, Portugal \\ E-mail: miguelpgouveia@gmail.com
}

How to cite this article: Pinto-Gouveia M, Gouveia AI, Brinca A, Vieira R. Tunneled island flaps in facial defects reconstruction. An Bras Dermatol. 2017;92(5 Suppl 1): 151-3. 